Pacific Journal of Mathematics

ON AN ADDITIVE ARITHMETIC FUNCTION

swami Alladi and Paul Erdós 


\title{
ON AN ADDITIVE ARITHMETIC FUNCTION
}

\author{
K. Alladi ANd P. ERdös
}

We discuss in this paper arithmetic properties of the function $A(n)=\sum_{p^{\alpha} \mid{ }_{n}} \alpha p$. Asymptotic estimates of $A(n)$ reveal the connection between $A(n)$ and large prime factors of $n$. The distribution modulo 2 of $A(n)$ turns out to be an interesting study and congruences involving $A(n)$ are considered. Moreover the very intimate connection between $A(n)$ and the partition of integers into primes provides a natural motivation for its study.

o. Introduction. Let a positive integer $n$ be expressed as a product of distinct primes in the canonical fashion $n=\prod_{i=1}^{r} p_{i}^{\alpha_{i}}$. Define a function $A(n)=\sum_{i=1}^{r} \alpha_{i} p_{i}$.

(i) The function $A(n)$ is not injective. In fact for a fixed integer $m$, the number of solutions in $n$ to $A(n)=m$, is the number of partitions of $m$ into primes.

(ii) $A(n)$ fluctuates in size appreciably. It is easily seen that $A(n)=n$ when $n$ is a prime, while $A(n)=O(\log n)$ when $n$ is a power of a small prime. Actually the "average order" of $A(n)$ turns out (as a corollary to Theorem 1.1) to be $\pi^{2} n / 6 \log n$. The term average order is defined below.

(iii) The function $A(n)$ is additive and one can expect it to take odd and even values with equal frequency.

The term "average order" calls for some explanation. We follow the usage in Hardy and Wright [6]. If $f(n)$ is a function defined on the positive integers we consider

$$
F(x)=\sum_{n \leqq x} f(n) .
$$

Usually $F$ can be expressed in terms of well behaved functions like polynomials or exponentials and the like. That is we seek an asymptotic estimate for $F$ in terms of these functions. Then we seek a similar well behaved function $g$ so that

$$
F(x)=\sum_{n \leq x} f(n) \sim \sum_{n \leq x} g(n) .
$$

The function $g$ may be thought of as the average order of $f$. For instance if $\phi$ is the Euler function then

$$
F(x)=\sum_{n \leqq x} \varphi(n)=\frac{3 x^{2}}{\pi^{2}}+O(x \log x) \sim \sum_{n \leqq x} \frac{6 n}{\pi^{2}}
$$

so the average order of $\varphi(n)$ is $6 n / \pi^{2}$. 
It is surprising that the function $A(n)$ with such nice arithmetic properties has not been studied in detail. Besides the work of one of us (KA; [1]) some of the other references are [4], [7], [8], [9] and [10]. Of course the contents of this paper are different.

1. Average order estimates of $A(n)$. Here and in what follows the letter $p$ (with or without subscript) shall always denote a prime.

So let $n=\prod_{i=1}^{r} p_{i}^{\alpha_{i}}$ and let $\Omega(n)=\sum_{i=1}^{r} \alpha_{i}, \omega(n)=r$. It is a well known result of Hardy and Ramanujan [6] that both $\Omega(n)$ and $\omega(n)$ have average order $\log \log n$, which tells us that generally the majority or prime factors occur only once. Applying this idea to $A(n)$ one expects it to have the same average order as $A^{*}(n)=$ $\sum_{i=1}^{r} p_{i}$. In this sum it is natural to believe that the largest prime factor of $n\left(P_{1}(n)\right.$ say) dominates the others so that $A(n)$ and $P_{1}(n)$ have the same average order. In fact this can be deduced as a corollary to Theorem 1.1 (where we prove much more) and the average order of $A(n)$ is $\pi^{2} n / 6 \log n$.

Let us assume without loss of generality that $p_{1}<p_{2}<\cdots<p_{r}$. Then let $P_{1}(n)=p_{r} ; P_{2}(n)=P_{1}\left(n / P_{1}(n)\right) ; P_{3}(n)=P_{1}\left(n / P_{1}(n) P_{2}(n)\right)$, etc., and in general

$$
P_{k}(n)=\left\{\begin{array}{l}
P_{1}\left(\frac{n}{P_{1}(n) \cdots P_{k-1}(n)}\right) \text { for } k \leqq \Omega(n) \\
0 \text { for } k>\Omega(n) .
\end{array}\right.
$$

Thus $P_{k}(n)$ is the $k$ th largest prime factor of $n$.

THEOREM 1.1. For all integers $m \geqq 1$ we have

$$
\sum_{n \leqq x} P_{m}(n) \sim \sum_{n \leqq x}\left\{A(n)-P_{1}(n)-\cdots-P_{m-1}(n)\right\} \sim \frac{k_{m} x^{1+(1 / m)}}{(\log x)^{m}}
$$

where $k_{m}>0$ is a constant depending only on $m$, and is a rational multiple of $\zeta(1+1 / m)$ where $\zeta$ is the Riemann Zeta function.

LemMA 1.2. If $s>1$ and $x$ a large real number then

$$
\sum_{p \geqq x} \frac{1}{p^{s}}=\frac{1}{(s-1) x^{s-1}(\log x)}+O\left(\frac{1}{x^{s-1}(\log x)^{2}}\right) .
$$

Proof. The proof of Lemma 1.2 is given by a simple direct method of using Stieltjes integrals, integration by parts and the prime number theorem in the form

$$
\pi(x)-l i(x)=O\left(\frac{x}{\log ^{\delta} x}\right)
$$


for all $\delta \geqq 2$. We have

$$
\begin{aligned}
\sum_{p \geqq x} \frac{1}{p^{s}}= & \int_{x^{-}}^{\infty} \frac{d \pi(y)}{y^{s}}=\int_{x^{-}}^{\infty} \frac{d y}{y^{s} \log y}+\int_{x^{-}}^{\infty} \frac{d\{\pi(y)-l i(y)\}}{y^{s}} \\
= & \int_{x^{-}}^{\infty} \frac{d y}{y^{s} \log y}+O\left(\frac{1}{x^{s-1} \log ^{\tilde{o}} x}\right) \\
& +\int_{x}^{\infty}\{\pi(y)-l i(y)\} O\left(\frac{1}{y^{s+1}}\right) d y
\end{aligned}
$$

for $\delta \geqq 2$ because of (1.1). Now

$$
\begin{aligned}
\int_{x}^{\infty} \frac{\{\pi(y)-l i(y)\} d y}{y^{s+1}} & =O\left(\int_{x}^{\infty} \frac{d y}{y^{s} \log ^{\delta} y}\right) \\
& =O\left(\frac{1}{x^{s-1} \log ^{\delta} x}\right) .
\end{aligned}
$$

So (1.3) and (1.2) give

$$
\sum_{p \leqq x} \frac{1}{p^{s}}=\int_{x}^{\infty} \frac{d y}{y^{s} \log y}+O\left(\frac{1}{x^{s-1} \log ^{2} x}\right) .
$$

But then

$$
\begin{aligned}
\int_{x}^{\infty} \frac{d y}{y^{s} \log y} & =\frac{1}{(s-1) x^{s-1} \log x}+O\left(\int_{x}^{\infty} \frac{d y}{y^{s} \log ^{2} y}\right) \\
& =\frac{1}{(s-1) x^{s-1} \log x}+O\left(\frac{1}{x^{s-1} \log ^{2} x}\right) .
\end{aligned}
$$

Clearly (1.5) and (1.4) prove Lemma (1.2)

The above lemma establishes the following result which will be used often in the proof of Theorem 1.1.

Lemma 1.3. Let $m$ be a positive integer and $s>1, r \geqq 1$ be given real numbers. Then for $x$ and $z$ sufficiently large real numbers with $x^{1+(1 / m)}<z<x^{3 m}$ we have

$\sum_{x \leqq p \leqq z / 2} \frac{1}{p^{s} \log ^{r}(z / p)}=\frac{1}{(s-1) x^{s-1} \log x \log ^{r}(z / x)}+O\left(\frac{\log \log x}{x^{s-1} \log ^{2} x \log ^{r}(z / x)}\right)$.

Proof. We break up the range of summation as

$$
\begin{aligned}
& \sum_{x \leqq p \leqq x \log ^{B} x} \frac{1}{p^{s} \log ^{r}(z / p)}+\sum_{x \log ^{B} x<p \leqq z / 2} \frac{1}{p^{s} \log ^{r}(z / p)} \\
& \quad=\sigma_{1}+\sigma_{2} \text { respectively }
\end{aligned}
$$

where $B$ for the moment is a constant not specified. Now in $\sigma_{1}$, $\log (z / p)=\log (z / x)+O(\log \log x)$ so that 


$$
\frac{1}{\log ^{r}(z / p)}=\frac{1}{\log ^{r}(z / x)}+O\left(\frac{\log \log x}{\log x \cdot \log ^{r}(z / x)}\right)
$$

because $\log (z / x)$ and $\log x$ are of the same order of magnitude. Now the above estimate, together with Lemma 1.2 gives

$$
\sigma_{1}=\frac{1}{(s-1) x^{s-1} \log x \log ^{r}(z / x)}+O\left(\frac{\log \log x}{x^{s-1} \log ^{2} x \log ^{r}(z / x)}\right) .
$$

To estimate $\sigma_{2}$ again apply Lemma 1.2 to get

$$
\sigma_{2}=O\left(\sum_{x \log B_{x<p}} \frac{1}{p^{s}}\right)=O\left(\frac{1}{x^{s-1} \log x \cdot \log ^{(s-1) B} x}\right) .
$$

Comparing $\sigma_{1}$ and $\sigma_{2}$ we note that by a suitable choice of $B$ Lemma 1.3 is true.

The crucial point in Lemma 1.3 is that by choice of $z, \log (z / x)$ and $\log x$ are of the same order of magnitude.

An argument similar to Lemma 1.2 yields the following:

LEMMA 1.4. If $s, r \geqq 0$, then

$$
\sum_{p \leqq x} \frac{p^{s}}{(\log p)^{r}}=\frac{x^{s+1}}{(s+1)(\log x)^{r+1}}+O\left(\frac{x^{s+1}}{\log ^{r+2} x}\right) .
$$

We omit the proof of Lemma 1.4, since it is similar to Lemma 1.2. Here we have to consider

$$
\sum_{p \leqq x} \frac{p^{s}}{\log ^{r} p}=\int_{2^{-}}^{x^{+}} \frac{y^{s} d \pi(y)}{\log ^{r} y}
$$

and compute just as we did in Lemma 1.2.

We now move on to the proof of Theorem 1.1. The proof involves complicated estimates in several places and we shall elaborate in detail the more important ones.

Proof of Theorem 1.1. We are first going to estimate $\sum_{n \leqq x} P_{m}(n)$. Let an integer $n$ be written as $n=k p_{1} \cdots p_{m}, p_{1} \leqq p_{2} \leqq \cdots \leqq p_{m}$, $P_{1}(k) \leqq p_{1}$, and let

$$
k=P_{m}^{*}(n)=\frac{n}{P_{1}(n) \cdots P_{m}(n)} .
$$

We keep $k=P_{m}^{*}(n)$ fixed and ask for those $n \leqq x$ for which $P_{m}^{*}(n)=k$. We sum $P_{m}(n)$ over these $n$ and finally sum over $k$. Actually only small values of $k$ will contribute to the principal term and large values will be treated separately.

So let $k$ be small. The word "small" will be explained below. 
Note that each $p_{i}$ can range from $P_{1}(k)$ up to the minimum of $p_{i+1}$ and $x / k p_{m} \cdots p_{i+1}$. So we shall break up the range of $p_{i+1}$, and discuss several cases, and in each of them we shall be able to decide without ambiguity which of $p_{i+1}$ and $x / k p_{m} \cdots p_{i+1}$ is smaller, thereby determining the range of $p_{i}$.

Case 1. Let $p_{m} \leqq \sqrt[m]{x / k}$. Here the range of $p_{i}$ is between $P_{1}(k)$ and $p_{i+1}$ for $i=1,2, \cdots, m-1$.

Case 2. Now let $\sqrt[m]{x / k}<p_{m} \leqq x / k$. We have now several choices. First we make $p_{m-1} \leqq \sqrt[m-1]{x / k p_{m}}$. Then the $p_{i}$ range from $P_{1}(k)$ to $p_{i+1}$ for $i=1,2, \cdots, m-2$.

Case 3. Here $\sqrt[m]{x / k}<p_{m} \leqq x / k$ and $\sqrt[m-1]{x / k p_{m}}<p_{m-1} \leqq p_{m}$. Here we make $p_{m-2} \leqq \sqrt[m-2]{x / k p_{m} p_{m-1}}$ so that $p_{i} \leqq p_{i+1}$ for $i=1,2, \cdots, m-3$.

General case. We have $\sqrt[m]{x / k}<p_{m} \leqq x / k, \sqrt[m-1]{x / k p_{m}}<p_{m-1} \leqq p_{m}$, $\sqrt[m-2]{x / k p_{m} p_{m-1}}<p_{m-2} \leqq p_{m-1} \cdots \sqrt[i+1]{x / k p_{m} \cdots p_{i+2}}<p_{i+1} \leqq p_{i+2} \quad$ with $p_{i} \leqq \sqrt[i]{x / k p_{m} \cdots p_{\imath+1}}$ so that $p_{i-1} \leqq p_{i}, p_{i-2} \leqq p_{i-1}, \cdots, p_{2} \leqq p_{3}$ and $p_{i} \leqq p_{2}$.

... etc.

So we have a total of $m$ cases to consider. We sum these over $k \leqq x^{\varepsilon}, \varepsilon=1 / m(m+1)$ and one can check that the contribution of $P_{1}(k)$ to each summation is negligible and so we omit writing it. We elaborate this below.

$\left(S_{m}\right)$

$$
\sum_{k<x^{\varepsilon}} \sum_{p_{m} \leqq \sqrt[m]{x / k}} \sum_{p_{m-1} \leqq p_{m}} \cdots \sum_{p_{2} \leqq p_{3}} \sum_{p_{1} \leqq p_{2}} p_{1}
$$

$\left(S_{m-1}\right) \quad \sum_{k<x^{\varepsilon}} \sum_{\sqrt[m]{x / k<p_{m} \leqq x / k}} \sum_{p_{m-1} \leq \sqrt[m]{-1} \sqrt[1]{x / k p_{m}}} \sum_{p_{m} \leq p_{m-1}} \cdots \sum_{p_{2} \leqq p_{3}} \sum_{p_{1} \leqq p_{2}} p_{1}$

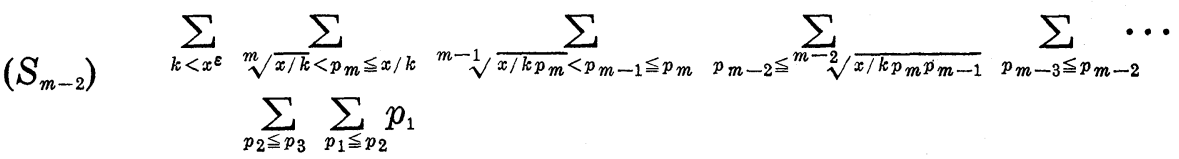

\section{$\cdots$}

$\cdots$

General term.

$$
\sum_{k<x \varepsilon} \sum_{\sqrt[m]{x / k}<p_{m} \leqq x / k} \sum_{\sqrt[m-1]{\sqrt[x / k p_{m}]{p_{p}}} p_{m-1} \leqq p_{m}} \sum_{m-2 \sqrt{x / k p_{m} p_{m-1}}<p_{m-2} \leq p_{m-1}} \cdots
$$




$$
\begin{aligned}
& \sum_{\sqrt[i+1]{x / k p_{m} \cdots p_{i+2}}<p_{i+1} \leqq p_{i+2}} \\
& \sum_{p_{i} \leqq i \sqrt{x / k p_{m} \cdots p_{i+1}}} \sum_{p_{i-1} \leqq p_{i}} \cdots \sum_{p_{2} \leqq p_{3}} \sum_{p_{1} \leqq p_{2}} p_{1}
\end{aligned}
$$

Last term.

$$
\begin{aligned}
& \sum_{k<x^{\varepsilon}} \sum_{\sqrt[m]{x / k}<p_{m} \leqq x / k} \sum_{\sqrt[m-1]{\sqrt[x / k p_{m}]{2}<p_{m-1} \leqq p_{m}}} \sum_{\sqrt[m-2]{\sqrt[x / k p_{m} p_{m-1}]{ }<p_{m-2} \leqq p_{m-1}}} \cdots \\
& \sum_{\sqrt{x / k p_{m} \cdots p_{3}<p_{2} \leqq p_{3}}} \sum_{p_{1} \leqq x / k p_{m} \cdots p_{2}} p_{1} \text {. }
\end{aligned}
$$

We shall first obtain upper bound estimates for each $\left(S_{i}\right)$. Our process will indicate how the terms grow and establishing the upper bound first makes explanation later simpler when we take up asymptotic estimates and need upper bound estimates for errors.

We know from Lemma 1.4 that $\sum_{p_{1} \leqq p_{2}} p_{1}=O\left(p_{2}^{2} / \log p_{2}\right)$. Now another application of Lemma 1.4 gives

$$
\sum_{p_{2} \leqq p_{3}} \sum_{p_{1} \leqq p_{2}} p_{1}=O\left(\frac{p_{3}^{3}}{\log ^{2} p_{3}}\right) \text {. }
$$

Thus taking the first $i$ summations in $\left(S_{i}\right)$ gives a term

$$
\begin{gathered}
O\left(\frac{x^{1+(1 / i)}}{\left(k p_{m} \cdots p_{i+1}\right)^{1+(1 / i)} L\left(i, i, x / k p_{m} \cdots p_{i+1}\right)}\right) \\
\text { where } L(i, j, x)=(\log \sqrt[i]{x})^{j}
\end{gathered}
$$

We have to sum the term above over the variable $p_{i+1}$ in the range $\sqrt[i+1]{x / k p_{m} \cdots p_{i+2}}<p_{i+1} \leqq p_{i+2}$. This is certainly less than if it is summed in the interval $\sqrt[i+1]{x / p_{m} \cdots p_{i+2}}<p_{i+1}$.

We are going to apply Lemma 1.3 with $z=x / k p_{m} \cdots p_{i+2}$ and $x$ in lemma replaced by $\sqrt[i+1]{x / k p_{m} \cdots p_{i+2}}$ which we will denote for the moment by $X$. We can also assume $z>X \log ^{\delta} X$, where $\delta>0$ is a suitably chosen large positive constant so that Lemma 1.3 is applicable. For if $z<X \log ^{\delta} X$ then we infer that

$$
p_{1} \leqq \frac{n}{k p_{m} \cdots p_{i+2}} \leqq \frac{x}{k p_{m} \cdots p_{i+2}}=O\left(\log ^{\delta^{\prime}} x\right)
$$

so that the sum of $P_{m}(n)$ for $n \leqq x$ over $n$ satisfying the above inequality is $O\left(x \log ^{\delta \prime} x\right)$ which is certainly of lower order of magnitude compared to the leading term mentioned in the theorem. We shall meet this situation as we move left along each summation and so we assume that $p_{1}>\log ^{\delta} x$ for some $\delta>0$, fixed and large, say $>m^{3}$. Now we apply Lemma 1.3 to infer from (1.6) that we get a term 
of size

$$
O\left(\frac{x^{1+(1 / i+1)}}{\left(k p_{m} \cdots p_{i+2}\right)^{1+(1 / i+1)} L\left(i+1, i+1, x / k p_{m} \cdots p_{i+2}\right)}\right) .
$$

Note that the term in (1.7) is just the term in (1.8) with $i$ replaced by $i+1$. Thus making the first $m$ summations of $\left(S_{i}\right)$ gives

$$
O\left(\frac{x^{1+(1 / m)}}{k^{1+(1 / m)} L(m, m, x / k)}\right)
$$

Obviously (1.8) summed over $k$ gives $O\left(x^{1+(1 / m)} / \log ^{m} x\right)$.

Now we proceed to the asymptotic estimate. We shall see that the leading terms we get are exactly those mentioned above. But the error terms can be estimated just as we got upper bound estimates but there will be an extra factor of $\log x$ in the denominator, giving a sum of lower order compared to the leading term.

So by Lemma 1.4,

$$
\sum_{p_{1} \leq p_{2}} p_{1}=\frac{p_{2}^{2}}{2 \log p_{2}}+O\left(\frac{p_{2}^{2}}{\log ^{2} p_{2}}\right)
$$

Summing the term in (1.9) up to $p_{3}$, we have by Lemma 1.4

$$
\sum_{p_{2} \leq p_{3}} \sum_{p_{1} \leq p_{2}} p_{1}=\frac{p_{3}^{3}}{6 \log ^{2} p_{3}}+O\left(\frac{p_{3}^{3}}{\log ^{3} p_{3}}\right) \text {. }
$$

So it is now clear that making the first $i$ summations as we did in (1.9) and (1.10) above, by repeating application of Lemma 1.4 we get

$$
\begin{aligned}
& \frac{x^{1+(1 / i)}}{(i+1) !\left(k p_{m} \cdots p_{i+1}\right)^{1+(1 / i)} L\left(i, i, x / k p_{m} \cdots p_{i+1}\right)} \\
& +O\left(\frac{x^{1+(1 / i)} \log \log x}{\left(k p_{m} \cdots p_{i+1}\right)^{1+(1 / i)} L\left(i, i+1, x / k p_{m} \cdots p_{i+1}\right)}\right)
\end{aligned}
$$

Now the $O$-term in (1.11) has an extra factor of log in the denominator, compared to the leading term. So summing this the way we did (1.6) up to (1.8) we get a term or order $O\left(x^{1+(1 / m)} \cdot \log \log x / \log ^{m+1} x\right)$. So we can forget the error term in (1.11).

Now each summation after the $i$ th summation in $\left(S_{i}\right)$ is of the form $\sum_{A<p_{j} \leq B}$ which we will interpret as $\sum_{A<p_{j}}-\sum_{B<p_{j}}$. There is no harm in writing it in this way, for each sum is actually a finite one because the $p_{j}$ 's occur in the denominator in the $i$ th summation. Now we apply Lemma 1.3 to estimate the sum of the leading term in (1.11) over the $(i+1)$ th summation. We have 


$$
\begin{aligned}
& \sqrt[i+1, \sqrt{x / k p_{m} \cdots p_{i+2}<p_{i+1} \leqq p_{i+2}}]{ } \frac{x^{1+(1 / i)}}{(i+1) !\left(k p_{m} \cdots p_{i+1}\right)^{1+(1 / i)} L\left(i, i, x / k p_{m} \cdots p_{i+1}\right)} \\
& = \\
& \quad+\frac{i x^{1+(1 / i+1)}}{(i+1) !\left(k p_{m} \cdots p_{i+2}\right)^{1+(1 / i+1)} L\left(i+1, i+1, x / k p_{m} \cdots p_{i+2}\right)} \\
& \left.\quad+\frac{x^{1+(1 / i+1)} \cdot \log \log x}{\left(k p_{m} \cdots p_{i+2}\right)^{1+(1 / 2+1)} L\left(i+1, i+2, x / k p_{m} \cdots p_{i+2}\right)}\right) \\
&
\end{aligned}
$$

Equation (1.12) needs some explanation. The first two terms on the right are obtained by considering $\sum_{A<p_{j}}$. As regards $\sum_{B<p_{j}}$ we distinguish two cases. The first is when $B>A \log ^{\delta} A$ ( $\delta$ sufficiently large, say $\left.>m^{2}\right)$. Now by Lemma 1.3 this sum is small compared to the former and there is no harm in writing it in the form of the third term on the right in (1.12). If $A<B<A \log ^{\delta} A$, then the log term does not change appreciably and again Lemma 1.3 gives the third term on the right of (1.12) as the leading term with the error being absorbed in the $O$-term in (1.12). Note that the $O$-term in (1.12) again has an extra factor of log in the denominator which as mentined before is pulled through to give an error term $O\left(x^{1+1 / m} \log \log x / \log ^{m+1} x\right)$. So what we are essentially saying is that we can forget the error terms totally since (1.12) is the type of estimate we will meet as we proceed left along $\left(S_{i}\right)$. As regards the leading terms, they will be of the form of the first term in (1.12) or the last term, depending whether we choose the left side bound which we call $A$, or the right side bound which we call $B$ in each summation. However in the summation involving $p_{m}$, we have to take $\sum_{\sqrt[m]{x / k}<p_{m}<\infty}$ because $\sum_{x / k<p_{m}<\infty}$ is a summation over the null set since $k p_{1} \cdots p_{m} \leqq x$. So in the first $j_{1}$ summations from the $i$ th one of $\left(S_{i}\right)$, we choose the left limit, and in the next $i_{1}$ we choose the right one, and in the next $j_{2}$ we choose the left one and so on. Then the sign of the total summation is $(-1)^{i_{1}+i_{2}+\cdots}$. Note that $j_{1}$ could be zero. We elaborate this below and this is our final step. The vertical lines in (1.13) tell us where the changes in limit takes place, and the arrow indicates the first step where we change.

$$
\left.\sum_{k<\alpha_{\varepsilon}} \sum_{\sqrt[m]{x \mid k<p_{m}<\infty}} \cdots\right|_{p_{i+j_{1}+i_{1}+1}<p_{i+j_{1}+i_{1}<\infty}} \cdots \sum_{p_{i+j_{1}+2}<p_{i+j_{1}+1}<\infty} \longleftarrow
$$

$$
\begin{aligned}
& \left|\sqrt[i+j_{1}]{\sqrt{x / k p_{m} \cdots p_{i}+j_{1}+1}<p_{i}+j_{1}<\infty}\right| \cdots \\
& \sum_{\sqrt[i+1]{x / k p_{m} \cdots p_{i+2}<p_{i+1}<\infty}} \frac{x^{1+(1 / i)}}{(i+1) !\left(k p_{m} \cdots p_{i+1}\right)^{1+(1 / i)} L\left(i, i, x / k p_{m} \cdots p_{i+1}\right)}
\end{aligned}
$$

The first summation in (1.13) gives the first term on the right of 
(1.12). What we are summing in (1.13) is the term in (1.11). In the process of going from (1.11) to (1.12) note that what has happened is that $i$ has been replaced by $i+1$ for the variables and there is an extra factor of $i$. So making the first $j_{1}$ summations we get a term which is

$$
\frac{i(i+1) \cdots\left(i+j_{1}-1\right) x^{1+\left(1 / i+j_{1}\right)}}{(i+1) !\left(k p_{m} \cdots p_{i+j_{1}+1}\right)^{1+\left(1 / i+j_{1}\right)} L\left(i+j_{1}, i+j_{1}, x / k p_{m} \cdots p_{j+j_{1}+1}\right)} .
$$

We have to sum the term in (1.14) over the variable $p_{i+j_{1+1}}$ in the summation indicated by the arrow above. Now by Lemma 1.3 we get a term of the type of the third term in (1.12) with $i$ replaced by $i+j_{1}$. So we have

$$
\frac{i(i+1) \cdots\left(i+j_{1}\right) x^{1+\left(1 / i+j_{1}\right)}}{\left(\begin{array}{c}
(i+1) !\left(k p_{m} \cdots p_{i+j_{1}+3}\right)^{1+\left(1 / i+j_{1}\right)}\left(p_{i+j_{1}+2}\right)^{1+\left(2 / i+j_{1}\right)} \\
\times L\left(i+j_{1}+1, i+j_{1}+1, x / k p_{m} \cdots p_{i+j_{1}+2}\right)
\end{array}\right)}
$$

The only thing we have to observe in (1.15) is that the exponent of $p_{i+j_{1}+2}$ is $1+\left(2 / i+j_{1}\right)$, and the exponent of $x$ has not changed from (1.14) to (1.15). This affects the nature of the constant to appear in the numerator of (1.16) below. What we get after the next summation is

$$
\frac{\left(\frac{i+j_{1}}{2}\right)(i)(i+1) \cdots\left(i+j_{1}\right) x^{1+\left(1 / i+j_{1}\right)}}{\left(\begin{array}{c}
(i+1) !\left(k p_{m} \cdots p_{i+j_{1}+4}\right)^{1+\left(1 / i+j_{1}\right)}\left(p_{i+j_{1}+3}\right)^{1+\left(3 / i+j_{1}\right)} \\
\times L\left(i+j_{1}+2, i+j_{1}+2, x / k p_{m} \cdots p_{i_{+j}+3}\right)
\end{array}\right)} .
$$

Now (1.16) is the term in (1.15) with subscripts changed by 1 and change of constants. So going to the end of $j_{1}+i_{1}$ summations we get

$$
\frac{\left(\frac{i+j_{1}}{2}\right) \cdot\left(\frac{i+j_{1}}{3}\right) \cdots\left(\frac{i+j_{1}}{i_{1}}\right)(i)(i+1) \cdots\left(i+j_{1}\right) x^{1+\left(1 / i+j_{1}\right)}}{\left(\begin{array}{c}
(i+1) !\left(k p_{m} \cdots p_{i+j_{1}+i_{1}+2}\right)^{1+\left(1 / i+j_{1}\right)}\left(p_{i+j_{1}+1}\right)^{1+\left(i_{1}+1 / i+j_{1}\right)} \\
\times L\left(i+j_{1}+i_{1}, i+j_{1}+i_{1}, x / k p_{m} \cdots p_{i+j_{1}+i_{1}+1}\right)
\end{array}\right)}
$$

Now when we sum (1.17) we are doing it in the range $A<p<\infty$, where $A$ is a left limit. If we show that this summation leads to a term similar to the one with which we started in (1.13) we are done. It is indeed remarkable that this happens. For again by Lemma 1.3 if we observe that

$$
1+\frac{1}{i+j_{1}}-\frac{i_{1}+1}{\left(i+j_{1}\right)\left(i+j_{1}+i_{1}+1\right)}=1+\frac{1}{i+j_{1}+i_{1}+1}
$$


we find that the exponent of $x$ which had remained constant for these $i_{1}$ summations changes suitably to give a term

$$
\frac{(\text { constant }) \cdot x^{1+1 /\left(i+j_{1}+i_{1}+1\right)}}{\left(\begin{array}{c}
\left(k p_{m} \cdots p_{i+j_{1}+i_{1}+2}\right)^{1+1 /\left(i+j_{1}+i_{1}+1\right)} \\
\times L\left(i+j_{1}+i_{1}+1, i+j_{1}+i_{1}+1, x / k p_{m} \cdots p_{i+j_{1}+i_{1}+2}\right)
\end{array}\right)} .
$$

Now the term in (1.18) is just the term in (1.13) with $i$ replaced by $i+j_{1}+i_{1}+1$. So after $i_{1}+j_{1}+1$ steps we are back in the same situation. So everytime we choose a left bound in a summation, we are back to the situation with which we started. But in the last summation involuing $p_{m}$, we have to choose the left bound. So ultimately we get

$$
\frac{c_{0} x^{1+(1 / m)}}{k^{1+(1 / m)} \log ^{m}(x / k)}
$$

where $c_{0}$ is rational. Summing this over $k<x^{\varepsilon}$, using a method similar to $\sigma_{1}$ and $\sigma_{2}$ in Lemma 1.3, gives

$$
\frac{c_{0} \zeta(1+(1 / m)) x^{1+(1 / m)}}{(\log x)^{m}} .
$$

Of course this is just one of the subcases of $\left(S_{i}\right)$. Considering all the subcases of $\left(S_{i}\right)$ we get $c_{i}^{*}$ rational and

$$
\frac{c_{i}^{*} \zeta\left(1+\frac{1}{m}\right) x^{1+(1 / m)}}{(\log x)^{m}} .
$$

Since the summations involve positive quantities we infer $c_{i}^{*}>0$. So summing over all the $i$ from 1 up to $m$, gives a positive rational $c_{m}$ so that the contribution from $\left(S_{1}\right)$ up to $\left(S_{m}\right)$ is

$$
\frac{c_{m} x^{1+(1 / m)} \zeta\left(1+\frac{1}{m}\right)}{(\log x)^{m}} \text {. }
$$

So this is the contribution for $k<x^{\varepsilon}, \varepsilon>0$. If $k>x^{\varepsilon}$ then

$$
\sum_{\substack{1 \leq n \leq x \\ k>x^{\varepsilon}}} P_{m}(n)=O\left(\sum_{n \leqq x} n^{(1-\varepsilon) / m}\right)=O\left(\frac{x^{1+(1 / m)}}{x^{\varepsilon / m}}\right) .
$$

So (1.20) and (1.19) yield

$$
\sum_{n \leq x} P_{m}(n)=\frac{c_{m} \zeta\left(1+\frac{1}{m}\right) x^{1+(1 / m)}}{\log ^{m} x}+O\left(\frac{x^{1+(1 / m)} \log \log x}{\log ^{m+1} x}\right)
$$


as shown by our investigation of error terms. Our theorem will be proved if we show that

$$
\sum_{n \leqq x}\left\{A(n)-P_{1}(n)-P_{2}(n)-\cdots-P_{m}(n)\right\}=o\left(\sum_{n \leqq x} P_{m}(n)\right) .
$$

Observe that $\left\{A(n)-P_{1}(n)-P_{2}(n)-\cdots-P_{m}(n)\right\}=A\left(P_{m}^{*}(n)\right)=A(k)$ and $A(k) \leqq \Omega(k) P_{1}(k)=\Omega(k) P_{m+1}(n) \leqq n^{1 /(m+1)} \cdot \Omega(n)$. So

$$
\begin{aligned}
\sum_{n \leqq x}\left\{A(n)-P_{1}(n)-P_{2}(n)-\cdots-P_{m}(n)\right\} & \leqq \sum_{n \leqq x} n^{1 /(m+1)} \Omega(n) \\
& =O\left(x^{1 /(m+1)} \sum_{n \leqq x} \Omega(n)\right) \\
& =O\left(x^{1+(1 /(m+1)} \log \log x\right) \\
& =o\left(\sum_{n \leqq x} P_{m}(n)\right)
\end{aligned}
$$

because of (1.21). The proof of Theorem 1.1 is complete.

COROLlaRY. The average order of $A(n)$ is $\pi^{2} n / 6 \log n$.

Proof. Set $m=1$ in Theorem 1.1. Then there is only one case to consider, namely $\left(S_{m}\right)=\left(S_{1}\right)$. So

$$
\sum_{n \leq x} A(n) \sim \sum_{n \leq x} P_{1}(n) \sim \frac{\pi^{2} x^{2}}{12 \log x}
$$

which gives the corollary.

It is clear that $A(n) \geqq A^{*}(n) \geqq P_{1}(n)$ so that $A^{*}(n)$ also has average order $\pi^{2} n / 6 \log n$.

THEOREM 1.5. The average order of $A(n)-A^{*}(n)$ is $\log \log n$. To be more precise

$$
\sum_{n \leqq x}\left\{A(n)-A^{*}(n)\right\}=x \log \log x+O(x) .
$$

Proof. It is not difficult to see that

$$
\sum_{n \leqq x}\left\{A(n)-A^{*}(n)\right\}=\sum_{p^{2} \leqq x} p\left[x / p^{2}\right]+\sum_{p^{3} \leqq x} p\left[x / p^{3}\right]+\cdots
$$

For if we write $A(n)-A^{*}(n)=\sum_{p^{\alpha}|| n}(\alpha-1) p$, then $p$ is counted $\left[x / p^{2}\right]$ more times, giving the first term. If $(\alpha-1) p=2 p$ we count only $\left[x / p^{3}\right]$ more times and so on. Now

$$
\begin{aligned}
\sum_{p^{2} \leqq x} p \frac{x}{p^{2}} & =\sum_{p \leqq \sqrt{x}} \frac{x}{p}+O\left(\sum_{p \leqq \sqrt{ } \bar{x}} p\right) \\
& =x \log \log x+O(x)
\end{aligned}
$$


and

$$
\sum_{p^{i} \leqq x} p \frac{x}{p^{i}}=x \sum_{p^{i} \leqq x} \frac{1}{p^{i-1}}=O\left(\sum_{p \leqq i \sqrt[i]{x}} p\right)
$$

So

$$
\sum_{i \geqq 3} \sum p \frac{x}{p^{i}}=O(x)
$$

which proves Theorem 1.5.

THEOREM 1.6. We have

$$
\sum_{n \leqq x}\left\{A^{*}(n)-P_{1}(n)-P_{2}(n)-\cdots-P_{m-1}(n)\right\} \sim \sum_{n \leqq x} P_{m}(n) \sim \frac{k_{m} x^{1+1 / m}}{(\log x)^{m}} .
$$

Proof. The theorem follows by combining Theorems 1.1 and 1.5.

THEOREM 1.7. For any fixed integer $M$, the set of solutions to $A(n)-A^{*}(n)=M$ has a natural density $>0$. (Note: A sequence $\left\{a_{n}\right\}_{n=1}^{\infty}$ has a natural density $\delta(A)$ if $\lim _{n \rightarrow \infty} n / a_{n}=\delta(A)$.)

Proof. Let us define an integer $n$ to be powerful if $n=\prod_{i=1}^{r} p_{i}^{\alpha_{i}}$, $\alpha_{i} \geqq 2, i=1,2, \cdots, r$. The set $S_{n}$ of integers of the form $n \cdot n^{\prime}$, $\left(n, n^{\prime}\right)=1$ and $n^{\prime}$ squarefree has natural density

$$
\begin{aligned}
\frac{1}{n} \prod_{p \nmid n}\left(1-\frac{1}{p}\right) \cdot \prod_{q \nmid n}\left(1-\frac{1}{q^{2}}\right) & =\frac{6}{n \pi^{2}} \prod_{p \nmid n} \frac{\left(1-\frac{1}{p}\right)}{\left(1-\frac{1}{p^{2}}\right)}=\frac{6}{n \pi^{2}} \prod_{p}\left(1+\frac{1}{p}\right)^{-1} \\
& =\delta\left(S_{n}\right) .
\end{aligned}
$$

Consider a partition of $M$ into primes as $M=\sum_{i=1}^{r} \beta_{i} p_{i}$. Any integer $n$ with $A(n)-A^{*}(n)=M$ is of the form $\prod_{i=1}^{r} p_{i}^{\left(\beta_{i}+1\right)} \Pi_{j=1}^{s} q_{j}$, where $q_{j}$ are primes different from $p_{i}$. Consider a particular partition $\pi_{j}$ of $M$, as $M=\sum_{i=1}^{r} \beta_{i} p_{i}$ and the powerful integer $m_{j}=\prod_{i=1}^{r} p_{i}^{\left(\beta_{i}+1\right)}$. This partition generates a set of solutions which is the set of numbers of the form $m_{j} \cdot m^{\prime},\left(m_{j}, m^{\prime}\right)=1, m^{\prime}$ square free. This set denoted by $S_{m_{j}}$ has natural density $\delta\left(S_{m_{j}}\right)$. Now the complete set of solutions is given by

$$
\bigcup_{j=1}^{p(M)} S_{m_{j}}
$$

where $p(M)$ is the number of partitions of $M$ into primes. Thus 


$$
\delta\left(\bigcup_{j=1}^{p(M)} S_{m_{j}}\right)=\sum_{j=1}^{p(M)} \delta\left(S_{m_{j}}\right)
$$

as $S_{m_{i}} \cap S_{m_{j}}=\varnothing$ if $i \neq j$. In fact because of (1.23) and (1.24) the density is a rational multiple of $1 / \zeta(2)=6 / \pi^{2}$.

2. Congruences involving $A(n)$. We now recall some results in [1]. For any integer $\mathrm{m}$, the number of solutions to $A(n)=m$ is the number of partitions of $m$ into primes. Note that $A(n)=n$ if and only if $n$ is a prime or $n=4$, so that it would be of interest to study the congruence

$$
n \equiv 0(\bmod A(n)) \text {. }
$$

Call a solution to (2.1) non-trivial if $n \neq A(n)$ and let the nontrivial solutions be called "special numbers". It is worth noting that if $m$ is fixed then the number of solutions to

$$
n \equiv 0(\bmod A(n)), \quad A(n)=m
$$

is the number of partitions of $m-A(m)$ into primes. So the number of solutions to (2.2) is much less than the number of solutions to $A(n)=m$, generally, and so one expects that special numbers are rather rare. Let $\left\{l_{n}\right\}$ denote the sequence of special numbers. The following can be proved (see [1]).

(1) The sequence $\left\{l_{n}\right\}$ is infinite.

(2) $\lim _{n \rightarrow \infty} A\left(l_{n}\right) / l_{n}=0$

(3) For any pair of integers $a$ and $b$, the number of solutions to $l_{n} \equiv a(\bmod b)$ is infinite.

(4) If $\pi(x, 2)$ represents the number of twin primes $\leqq x$ and $\pi(x, 2) \sim c x / \log ^{2} x$ then $\lim _{n \rightarrow \infty} l_{n} / l_{n+1}=1$.

Denote by $\mathscr{L}(x)$ the number of $l_{n} \leqq x$. We obtain bounds for $\mathscr{L}(x)$.

THEOREM 2.1. There exists a contant $c>0$ so that for all $x \geqq e$

$$
\mathscr{L}(x)=O\left(\frac{x}{e^{c \sqrt{\log x \log \log x}}}\right) .
$$

Proof. As before $P_{1}(n)$ denotes the largest prime factor of $n$. By a result of deBruijn (see [2], page 54, equation 1.6), if $\psi(x, y)$ is the number of solutions $\leqq x$ to $P_{1}(n)<y$ then

$$
\psi(x, y)<c_{1} x \log ^{2} y e^{-u \log u-\log \log u+c_{2} u}
$$

where $y=x^{1 / u}$. Now if we set $u=\sqrt{\log x / \log \log x}$, then $y$ is seen to be $e^{\sqrt{\log x \log \log x}}$. Also 


$$
\begin{aligned}
\psi(x, y) & <\frac{c_{1} x \log x \log \log x}{e^{1 / 2 \sqrt{\log x \log \log x-c_{3} u}}} \\
& =O\left(\frac{x}{e^{1 / 2 \sqrt{\log x \log \log x}}}\right) .
\end{aligned}
$$

So we will restrict our attention to $P_{1}(n)>e^{\sqrt{\log x \log \log x}}$ for the number of $n$ not satisfying this is given by (2.4). We also assume that if $\tau(n)$ is the number of divisors of $n$ then

$$
\tau(n)<e^{1 / 2 \sqrt{\log x \log \log x}} .
$$

For the number of integers not satisfying (2.5) is easily seen to be

$$
O\left(\frac{x \log x}{e^{1 / 2 \sqrt{\log x \log \log x}}}\right)
$$

becaúse $\sum \tau(n)=O(x \log x)$. So we confine ourselves to $n \leqq x$ satisfying (2.5) and $P_{1}(n)>e^{\sqrt{\log x \log \log x}}$. Let these numbers be denoted by the sequence $\left\{n_{i}\right\}$. Denote by $t$ the following

$$
\frac{n_{i}}{P_{1}\left(n_{i}\right)}=t \Longrightarrow A\left(n_{i}\right)=P_{1}\left(n_{i}\right)+A(t) \text {. }
$$

Clearly as $n_{i} \leqq x$ we have

$$
t<x e^{-\sqrt{\log x \log \log x}} .
$$

Let $t$ for the moment be fixed. We have two possibilities arising out of (2.7).

Case 1. $A(t) \equiv 0\left(\bmod P_{1}\left(n_{i}\right)\right)$.

Since $t$ is fixed and we are seeking solutions to (2.7) it is clear that the $P_{1}\left(n_{i}\right)$ are distinct and divide $A(t)$. Also as we require special numbers, $t \neq 1$ and so $A(t) \neq 0$. Thus the number of solutions to Case 1 is at most $O(\log x)$, since $t \leqq x$.

Case 2. $A(t) \not \equiv 0\left(\bmod P_{1}\left(n_{i}\right)\right)$.

Since we are interested in special numbers we require

$$
n_{i} \equiv 0\left(\bmod A\left(n_{i}\right)\right) \equiv o\left(\bmod P_{1}\left(n_{i}\right)+A(t)\right) .
$$

But Case 2 implies that $\left(A(t), P_{1}\left(n_{i}\right)\right)=1$ which means (2.9) gives

$$
\frac{n_{i}}{P_{1}\left(n_{i}\right)}=t \equiv 0\left(\bmod P_{1}\left(n_{i}\right)+A(t)\right) .
$$

Again $A(t)+P_{1}\left(n_{i}\right)$ are distinct when $t$ is fixed, so that by our choice of $n_{i}$, by (2.5) the number of solutions to (2.10) is less than 


$$
e^{1 / 2 \sqrt{\log x \log \log x}} .
$$

Thus for fixed $t$, the number of solutions to (2.7) in special numbers is at most

$$
\log x+e^{1 / 2 \sqrt{\log x \log \log x}}=O\left(e^{1 / 2 \sqrt{\log x \log \log x}}\right) .
$$

But by (2.8) we have an upper bound on the number of choices of $t$. Thus the $\left\{l_{n}\right\}$ among the $n_{i}$ do not exceed

$$
O\left(\frac{x}{e^{\sqrt{\log x \log \log x}}} e^{1 / 2 \sqrt{\log x \log \log x}}\right)=O\left(\frac{x}{e^{1 / 2 \sqrt{\log x \log \log x}}}\right) .
$$

But the number of integers not included among the $\left\{n_{i}\right\}$ is by (2.6) and (2.4)

$$
O\left(\frac{x \log x}{e^{1 / 2 \sqrt{\log x \log \log x}}}\right)
$$

So (2.12) and (2.11) prove the theorem with any $c<1 / 2$.

Now for a lower bound,

THEOREM 2.2. There exists a constant $c^{\prime}>0$ so that

$$
\mathscr{L}(x) \gg \frac{x}{e^{c^{\prime} \sqrt{\log x \log \log x}}}
$$

Proof. Let $x$ be a large real number and define $z$ and $k$ as follows:

$$
z=\frac{e^{\sqrt{\log x \log \log x}}}{(\log x)^{c_{4}}} ; \quad k=\sqrt{\frac{\log x}{\log \log x}}
$$

where $c_{4}>0$ is a constant to be determined soon. Consider the number of $k$-tuples of primes $\leqq z$ which clearly is $\left(\begin{array}{c}\pi(z) \\ k\end{array}\right)$. This can be easily seen to be greater than

$$
\begin{aligned}
\frac{(\pi(z)-k)^{k}}{k !} & >\frac{(\pi(z)-k)^{k}}{e^{k \log k}}>\left(\frac{e^{\sqrt{\log x \log \log x}}}{(\log x)^{c_{4}+\varepsilon} \sqrt{\overline{\log x \log \log x}}}\right)^{k} \cdot \frac{1}{e^{k \log k}} \\
& >\frac{x}{e^{\left(c_{4}+1+\varepsilon\right) \sqrt{\log x \log \log x}}}
\end{aligned}
$$

for sufficiently large $x$. Now let the product of these primes define a sequence $\left\{u_{j}\right\}$, all $\leqq z^{k}$ which is seen to be

$$
z^{k}=\frac{x}{e^{c_{4} \sqrt{\log x \log 1 \log x}}}
$$


Let us put $c_{5}=c_{4}+1+\varepsilon, \varepsilon>0$ arbitrary. So we have at least $x e^{-c_{5} \sqrt{\log x \log \log x}}$ distinct numbers $<z^{k}$ given in (2.15). Consider the product of the first $r$-primes $p_{1} \cdots p_{r}$ such that it is just greater than $z k^{2}$. We shall produce a number $L$ so that

$$
A\left(u_{j} p_{1} \cdots p_{r} \cdot L\right)=p_{1} \cdots p_{r}
$$

so that $u_{j} \cdot p_{1} \cdots p_{r} \cdot L$ is a special number. Clearly we need

$$
A(L)=p_{1} \cdots p_{r}-\left(p_{1}+p_{2}+\cdots+p_{r}\right)-A\left(u_{j}\right) .
$$

By our choice of $u_{j}, A\left(u_{j}\right) \leqq z k$ and so the quantity in (2.16) is of order $z k^{2}$. If it is odd, use Vinogradov's theorem, and partition it into three primes, and take their product to get $L$. Otherwise subtract $L$ and partition the rest into three primes and $L$ is the product of these primes. In any case $L=O\left(\left(z k^{2}\right)^{3}\right)$ and so $p_{2} \cdots p_{r} \cdot L=O\left(\left(z k^{2}\right)^{5}\right)$. Now we want $u_{j} \cdot p_{1} \cdots p_{r} \cdot L \leqq x$. So choose $c_{4}>5$ so that by (2.15) the product $u_{j} \cdot p_{1} \cdots p_{r} \cdot L \leqq x$. Now the number of repetitions among $u_{j} \cdot p_{1} \cdots p_{r} \cdot L$ is at most $\left(z k^{2}\right)^{4}$. So they are at least

$$
\frac{x}{e^{c_{5} \sqrt{1 \log x \log \log x}}} \cdot \frac{1}{\left(z k^{2}\right)^{4}} \gg \frac{x}{e^{(10+\varepsilon) \sqrt{\log x \log \log x}}}
$$

special numbers $\leqq x$, by our choice of $c_{4}$ and $z$. So Theorem 2.2 holds with any $c^{\prime \prime}>10+\varepsilon$.

REMARK. The problem discussed in this section can be worded differently. "How often can a sum of primes (not necessarily distinct) divide their product?" That is we want $\sum \alpha_{i} p_{i}$ to divide $\Pi p_{i}^{\alpha_{i}}$ where each $p_{i}$ has $\alpha_{i}$ repetitions. This is precisely of the problem of special numbers discussed above.

It might be true that $\mathscr{L}(x)$ is actually of the order

$$
x e^{-c^{\prime \prime}(1+o(1)) \sqrt{\log x \log \log x}} .
$$

An asymptotic formula for $\mathscr{L}(x)$ seems very hard to obtain. The constants in Theorems 2.1 and 2.2 can be sharpened with more accurate computation, but our estimates indicate the method.

We conclude this section with a few interesting questions. Does the product of consecutive primes infinitely often determine special numbers? For instance $2 \cdot 3 \cdot 5 \equiv 0(\bmod 2+3+5)$. Also $n=$ $2 \cdot 3 \cdot 5 \cdot 7 \cdot 11 \cdot 13 \cdot 17 \cdot 19$ is special. $A(n)$ is $77=7 \cdot 11$. Another example is $n=2 \cdot 3 \cdot 5 \cdot 7 \cdot 11 \cdot 13 \cdot 17 \cdot 19 \cdot 23 \cdot 29 \cdot 31 \cdot 37 \cdot 41$ where $A(n)=238=2 \cdot 7 \cdot 17$. We guess there are infinitely many such numbers!

It is easy to see that infinitely many special numbers are square free. For, take a prime $p$, and partition $p-2$ into distinct primes $p_{1}+p_{2}+\cdots+p_{r}$. This is possible. Then $2 \cdot p \cdot p_{1} \cdot p_{2} \cdot \cdots \cdot p_{r}$ is special 
for $A\left(2 \cdot p \cdot p_{1} \cdot p_{2} \cdot \cdots \cdot p_{r}\right)=2 p$.

One can show that for sufficiently large composite numbers $n$, there exists $m$ with $m \equiv 0(\bmod A(m)), A(m)=n$ and $m / n$ square free and prime to $n$. This follows from Vinogradov's theorem, and here we partition $n-A(n)$ into primes. It might be of interest to determine (besides the primes), all the other $n$ for which this is not true.

3. Distribution modulo 2. First we shall show that $A(n)$ is uniformly distributed modulo 2, and the error is of the order of the sum of the Möbius function $M(x)$. Here we shall concentrate on the function $\alpha(n)=(-1)^{A(n)}$, which is easily seen to obey $\alpha(m \cdot n)=$ $\alpha(m) \cdot \alpha(n) \forall m, n$. Thus for any complex number $s$, with $\operatorname{Re} s>1$, we have

$$
\sum_{n=1}^{\infty} \frac{\alpha(n)}{n^{s}}=\prod_{p}\left(1-\frac{\alpha(p)}{p^{s}}\right)^{-1}=\frac{2^{s}+1}{2^{s}-1} \cdot \frac{\zeta(2 s)}{\zeta(s)} .
$$

Now as $s \rightarrow 1^{+}$, the right side of (3.1) tends to zero, and so it is natural to expect

$$
\sum_{n=1}^{\infty} \frac{\alpha(n)}{n}=0
$$

We prove (3.2) in Theorem 3.2. But first we show that $A(n)$ is uniformly distributed modulo 2. This is expressed in

THEOREM 3.1. There exists a constant $c_{6}>0$ so that

$$
\sum_{1 \leqq n \leqq x} \alpha(n)=O\left(x e^{-c_{6} \sqrt{\log x \log \log x}}\right) \text {. }
$$

Proof. Consider the sum $a(n)=\sum_{d \mid n} \alpha(d)$. If $n=2^{\alpha_{0}} \prod_{i=1}^{r} p_{\imath}^{\alpha_{i}}$ where $p_{i}$ are odd, then

$$
\begin{aligned}
a(n) & =\left(\alpha_{0}+1\right) \prod_{i=1}^{r}\left(1+\alpha\left(p_{i}\right)+\alpha\left(p_{i}^{2}\right)+\cdots+\alpha\left(p_{i}^{\alpha}\right)\right) \\
& =\left(\alpha_{0}+1\right) \prod_{i=1}^{r} \frac{(-1)^{\left(\alpha_{i}+1\right) p_{i}}-1}{(-) 1^{p_{i}}-1}
\end{aligned}
$$

We infer from (3.3) that if any one of the $\alpha_{i}$ is odd, then $a(n)=0$. Thus $a(n)$ is non-zero only over integers of the form $2^{\alpha_{0}} \cdot m^{2}$ where $m$ is odd. Also $a(n) \geqq 0$. Clearly

$$
\sum_{n \leqq x} a(n) \leqq \sum_{2^{\alpha_{0}} \leqq x}\left(\alpha_{0}+1\right) \sqrt{x / 2^{\alpha_{0}}}=O(\sqrt{x})
$$




$$
\sum_{n=1}^{\infty} \frac{a(n)}{n}=c_{7}<\infty
$$

Now if $\mu$ is the Möbius function then

$$
\begin{aligned}
\sum_{1 \leqq n \leqq x} \alpha(n) & =\sum_{1 \leqq n \leqq x} \sum_{d \mid n} \mu(d) a\left(\frac{n}{d}\right) \\
& =\sum_{1 \leqq d \leqq \sqrt{x}} a(d) \sum_{1 \leqq d^{\prime} \leqq x / d} \mu\left(d^{\prime}\right)+\sum_{1 \leqq d<\sqrt{x}} \mu(d) \sum_{\sqrt{x}<d^{\prime} \leqq x^{\prime} / d} a\left(d^{\prime}\right) \\
& =\sum_{1 \leqq d \leqq \sqrt{x}} a(d) M\left(\frac{x}{d}\right)+\sum_{1 \leqq d \leqq \sqrt{x}} O(\sqrt{x / d}) \\
& =\sum_{1 \leqq d \leqq \sqrt{x}} a(d) M\left(\frac{x}{d}\right)+O\left(x^{3 / 4}\right)
\end{aligned}
$$

by using (3.4). It is known from the investigation of the error term in the prime number theorem (see [3]) that there is a constant $c_{8}>0$ so that

$$
M(x)=O\left(x e^{-c_{8} \sqrt{\log x \log \log x}}\right)
$$

so that one inferes from (3.6), (3.7), and (3.5) that Theorem 3.1 is true. Finally we prove

THEOREM 3.2. $\sum_{n=1}^{\infty} \alpha(n) / n=0$.

Proof. As we have already remarked, $a(n)$ is nonzero only at values $n=2^{\alpha_{0}} \cdot m^{2}$, where $m$ is odd, and $a(n)$ here is $\alpha_{0}+1$. Thus

$$
\begin{aligned}
\sum_{n>x} \frac{a(n)}{n} & =\sum_{\substack{\alpha_{0}=0 \\
m \text { odd }}}^{\infty} \sum_{\sqrt{x / 2^{\alpha} 0_{0}}} \frac{\alpha_{0}+1}{2^{\alpha_{0}} \cdot m^{2}} \\
& =\sum_{\alpha_{0}=0}^{\infty} \frac{\alpha_{0}+1}{2^{\alpha_{0}}} \cdot O\left(\sqrt{2^{\alpha_{0}} / x}\right)=O(1 / \sqrt{\bar{x}})
\end{aligned}
$$

so that if we set

$$
\chi(x)=x\left(\sum_{n>x} \frac{a(n)}{n}\right)
$$

we infer from (3.8) that

$$
\chi(x)=O\left(x^{1 / 2}\right) .
$$

Also $\chi$ is of bounded variation on finite intervals. It follows that

$$
\begin{aligned}
x \sum_{d \leq x} \frac{\alpha(d)}{d} & =x \sum_{n m \leqq x} \frac{\mu(m) a(n)}{m n} \\
& =x \sum_{m \leqq x} \frac{\mu(m)}{m} \sum_{n \leqq x / m} \frac{a(n)}{n} .
\end{aligned}
$$


Now by (3.5), (3.10) is rewritten as

$$
\begin{aligned}
x \sum_{d \leqq x} \frac{\alpha(d)}{d} & =c_{7} x \sum_{m \leqq x} \frac{\mu(m)}{m}-\sum_{m \leqq x} \mu(m) \frac{x}{m} \sum_{n>x / m} \frac{a(n)}{n} \\
& =c_{7} x \sum_{m \leqq x} \frac{\mu(m)}{m}-\sum_{m \leqq x} \mu(m) \chi\left(\frac{x}{m}\right) .
\end{aligned}
$$

We can deduce Theorem 3.2 from (3.11), if we appeal to Axer's Theorem 267 in [5] stated below.

AXER's THEOREM. If $\left\{b_{n}\right\}$ is a sequence of real numbers with

$$
\sum_{n \leq x} b_{n}=o(x) \quad \text { and } \quad\left|b_{n}\right|=O(1)
$$

and $\chi$ a function of bounded variation on finite intervals with $\chi(x)=O\left(x^{\alpha}\right)$ for some $0<\alpha<1$, then

$$
\sum_{n \leq x} b_{n} \chi\left(\frac{x}{n}\right)=o(x) .
$$

If we apply Axer's Theorem with $b_{n}=\mu(n)$, and observe that $\chi(x)=O\left(x^{\alpha}\right)$ with $\alpha=1 / 2$ in (3.9) then because

$$
\sum_{n=1}^{\infty} \frac{\mu(n)}{n}=0
$$

we infer from (3.11) that Theorem 3.2 is true. For a proof of (3.12) see [5].

By slight variation of the proofs of the above theorems one can show that for some fixed integer $N$

$$
\sum_{\substack{1 \leq n \leq x \\(n, N)=1}} \alpha(n)=O\left(x e^{-c_{9} \sqrt{\log x \log \log x}}\right)
$$

and

$$
\sum_{\substack{n=1 \\(n, N)=1}}^{\infty} \frac{\alpha(n)}{n}=0
$$

REMARK. We would like to conclude by mentioning a few interesting problems connected with $A(n)$.

Let $f(n)$ be the smallest integer $m$ so that $A(m)=n$. Consider a partition of $n$ into primes, $n=p_{1}+p_{2}+\cdots$ where $p_{1}$ is the largest prime $\leqq n, p_{1} \neq n-1, p_{2}$ the largest prime $\leqq n-p_{1}, p_{2} \neq n-p_{1}-1$, and so on, and denote by $\mathrm{F}(n)=p_{1} \cdot p_{2} \cdot \cdots$. It appears at first sight that $f(n)=F(n)$ but this need not be so. In fact this does not happen quite often. For instance $f(6)=8, F(6)=9$. It would be of 
interest to consider the relative sizes of $f(n)$ and $F(n)$.

In this context we mention the following curious problem. Replace the primes above by squares. That is

$$
G(n)=\min \quad \Pi \alpha_{i}^{2} ; g(n)=\Pi b_{i}^{2} \quad \sum \alpha_{i}^{2}=n
$$

where $b_{i}^{2}$ is the largest square $\leqq n$, and so on. It might be true that both $G(n)$ and $g(n)$ are both $<c \cdot n^{2}$ where $c$ is a constant. In $G(n)$ above, we require that not more than three of the $a_{i}=1$, for $3=1+1+1$ is the only decomposition of 3 .

For more results on $A(n)$, see a forthcoming paper of Erdös and Pomerance where it is proved that the set of solutions to $A(n)=$ $A(n+1)$ is of density zero. One could also consider equations involving $A(n)$ of similar type but these problems are in general difficult.

ACKNOWLEDGMENT. We are grateful to the referee for his comments and suggestions to improve the paper.

One of (K. A.) would like to express his gratitude to Professor E. G. Straus for the encouragement he gave and we would like to thank him for helping us in preparing the manuscript.

\section{REFERENCES}

1. K. Alladi, $A$ new logarithmic function, Srinivasa Ramanujan Commemmoration Volume, Oxford Press, Madras, India, (1974) part II.

2. N. J. deBruijn, On the number of positive integers $\leqq x$ free of prime factors $>y$, Indag. Math., (1951), 50-60.

3. K. Chandrasekharan, Arithmetical Functions, Springer-Verlag, 1970, 50-65.

4. L. M. Chawla, On a pair of Arithmetic functions, J. Natur. Sci. and Math., 8 (1968), 263-269.

5. G. H. Hardy, Divergent Series, Oxford, Clarendon Press, 1949, 378-380.

6. G H. Hardy and E. M. Wright, An Introduction to the Theory of Numbers, Oxford, Clarendon Press, 4-ed., 1968, 263, 354-358.

7. S. M. Kerawala, On a pair of Arithmetic functions analogous to Chawla's pair,

J. Natur. Sci. and Math., 9 (1969), 97-103.

8. M. O. LeVan, On the sum $F(x, r)$, J. Natur. Sci. and Math., 11 (1971), 285-290.

9. J. MohanLal, On the iterates of a number theoretic function, Math. Comp., 23 (1969), 181-183, A-34-38.

10. N. J. Nicolas, Sur-1-ordre maximum d'un element dans le groupe $S_{n}$ des permutations, Acta Arith., 14 (1958), 315-332.

Received October 29, 1975 and in revised form October 29, 1976.

UNIVERSITY OF CALIFORNIA

LOS ANGELES, CA 90024

AND

Hungarian ACADEMY OF SCIENCES

BUDAPEST, HUNGARY 


\section{PACIFIC JOURNAL OF MATHEMATICS}

\section{EDITORS}

RICHARD ARENS (Managing Editor)

University of California

Los Angeles, CA 90024

Charles W. Curtis

University of Oregon

Eugene, OR 97403

C. C. MOORE

University of California

Berkeley, CA 94720

\section{J. DugundJI}

Department of Mathematics

University of Southern California

Los Angeles, CA 90007

R. FinN and J. Milgram

Stanford University

Stanford, CA 94305

ASSOCIATE EDITORS
E. F. BECKENBACH
B. H. NeumanN
F. WOLF
K. YOSHIDA

\section{SUPPORTING INSTITUTIONS}

UNIVERSITY OF BRITISH COLUMBIA CALIFORNIA INSTITUTE OF TECHNOLOGY

UNIVERSITY OF CALIFORNIA

MONTANA STATE UNIVERSITY

UNIVERSITY OF NEVADA, RENO

NEW MEXICO STATE UNIVERSITY

OREGON STATE UNIVERSITY

UNIVERSITY OF OREGON

OSAKA UNIVERSITY

\author{
UNIVERSITY OF SOUTHERN CALIFORNIA \\ STANFORD UNIVERSITY \\ UNIVERSITY OF HAWAII \\ UNIVERSITY OF TOKYO \\ UNIVERSITY OF UTAH \\ WASHINGTON STATE UNIVERSITY \\ UNIVERSITY OF WASHINGTON \\ AMERICAN MATHEMATICAL SOCIETY
}

The Supporting Institutions listed above contribute to the cost of publication of this Journal, but they are not owners or publishers and have no responsibility for its content or policies.

Mathematical papers intended for publication in the Pacific Jaurnal of Mathematics should be in typed form or offset-reproduced, (not dittoed), double spaced with large margins. Please do not use built up fractions in the text of your manuscript. You may however, use them in the displayed equations. Underline Greek letters in red, German in green, and script in blue. The first paragraph or two must be capable of being used separately as a synopsis of the entire paper. Items of the bibliography should not be cited there unless absolutely necessary, in which case they must be identified by author and Journal, rather than by item number. Manuscripts, in triplicate, may be sent to any one of the editors. Please classify according to the scheme of Math. Reviews, Index to Vol. 39. All other communications should be addressed to the managing editor, or Elaine Barth, University of California, Los Angeles, California, 90024.

The Pacific Journal of Mathematics expects the author's institution to pay page charges, and reserves the right to delay publication for nonpayment of charges in case of financial emergency.

100 reprints are provided free for each article, only if page charges have been substantially paid. Additional copies may be obtained at cost in multiples of 50 .

The Pacific Journal of Mathematics is issued monthly as of January 1966. Regular subscription rate: $\$ 7200$ a year (6 Vols., 12 issues). Special rate: $\$ 36.00$ a year to individual members of supporting institutions.

Subscriptions, orders for back numbers, and changes of address should be sent to Pacific Journal of Mathematics, 103 Highland Boulevard, Berkeley, California, 94708.

PUBLISHED BY PACIFIC JOURNAL OF MATHEMATICS, A NON-PROFIT CORPORATION

Printed at Kokusai Bunken Insatsusha (International Academic Printing Co., Ltd.). 8-8, 3-chome, Takadanobaba, Shinjuku-ku, Tokyo 160, Japan.

Copyright (C) 1975 by Pacific Journal of Mathematics Manufactured and first issued in Japan 


\section{Pacific Journal of Mathematics}

\section{Vol. 71, No. $2 \quad$ December, 1977}

Krishnaswami Alladi and Paul Erdős, On an additive arithmetic

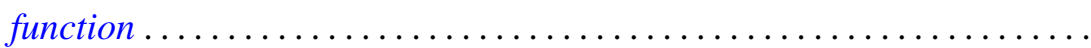

James Bailey and Dale Rolfsen, An unexpected surgery construction of a

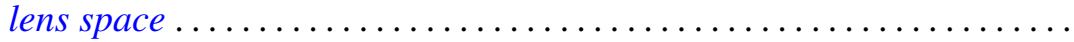

Lawrence James Brenton, On the Riemann-Roch equation for singular

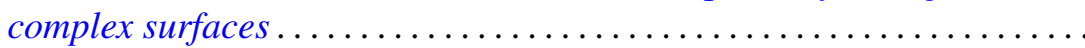

James Glenn Brookshear, Projective ideals in rings of continuous

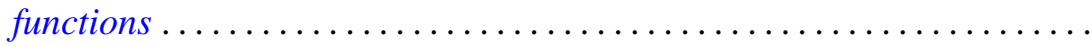

Lawrence Gerald Brown, Stable isomorphism of hereditary subalgebras of

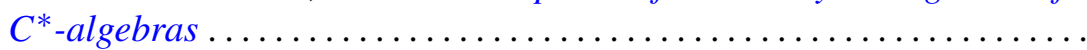

Lawrence Gerald Brown, Philip Palmer Green and Marc Aristide Rieffel, Stable isomorphism and strong Morita equivalence of $C^{*}$-algebras....

N. Burgoyne, Robert L. Griess, Jr. and Richard Lyons, Maximal subgroups

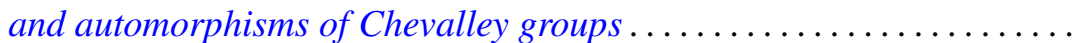
365

Yuen-Kwok Chan, Constructive foundations of potential theory .... 405

Peter Fletcher and William Lindgren, On $w \Delta$-spaces, $w \sigma$-spaces and

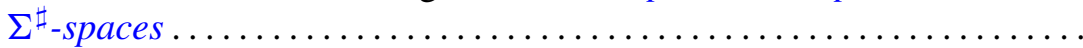

Louis M. Friedler and Dix Hayes Pettey, Inverse limits and mappings of

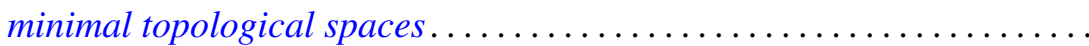

Robert E. Hartwig and Jiang Luh, A note on the group structure of unit regular ring elements.

I. Martin (Irving) Isaacs, Real representations of groups with a single involution ...

Nicolas P. Jewell, The existence of discontinuous module derivations . .

Antonio M. Lopez, The maximal right quotient semigroup of a strong semilattice of semigroups .......................

Dennis McGavran, $T^{n}$-actions on simply connected $(n+2)$-manifolds

Charles Anthony Micchelli and Allan Pinkus, Total positivity and the exact $n$-width of certain sets in $L^{1}$.

Barada K. Ray and Billy E. Rhoades, Fixed point-theorems for mappings with a contractive iterate .......................

Fred Richman and Elbert A. Walker, Ext in pre-Abelian categories. .

Raymond Craig Roan, Weak* generators of $H^{\infty}$ and $l^{1}$..

Saburou Saitoh, The exact Bergman kernel and the kernels of Szegö type...

Kung-Wei Yang, Operators invertible modulo the weakly compact 